Editorial

\title{
Skull Base Surgery in the 21st Century: Accelerated Revolution
}

\author{
Ricardo L. Carrau, MD ${ }^{1}$ \\ ${ }^{1}$ Department of Otolaryngology-Head \& Neck Surgery, \\ Comprehensive Skull Base Surgery Program, The Ohio State \\ University Wexner Medical Center, Columbus, Ohio, United States \\ Int Arch Otorhinolaryngol 2014;18:S121-S122.
}

All evolution in thought and conduct must first be perceived as heresy and misconduct. George Bernard Shaw

Technological developments in the 21st century are emerging at a neck-breaking pace, bringing dramatic changes to all aspects of our daily life at a global scale. Medicine and other components of health care have been positively affected by new capabilities regarding the gathering, interpretation, and storage of information-whether this is clinical, imaging, physiological, genetic, or other. In the process, some preexistent technologies have been enhanced and others have been completely replaced by novel devices.

As a result of these advances, we are witnessing the transition from intuitive or empirical diagnosis and treatment to a more exact and personalized practice of medicine, where physicians are no longer reacting to mere symptoms and signs but looking at the root of a disease and designing treatments specific to its pathogenesis. Diseases that in the past seemed identical, and hence received similar treatments but obtained disparate results, are now recognized as distinctly different problems. Better differentiation has led to customized treatments that afford superior and predictable outcomes.

Surgical specialties have benefitted also by the rapid evolution of established techniques, the development of novel procedures, and the rebirth of older methods brought back to the surgical armamentarium after being renovated by technological advances. This transformation has led to improved outcomes while minimizing morbidity.

Skull base surgery is an ideal milieu to integrate these changes. Uniquely posed as a model of interspecialty cooperation and cross-pollination by a surgical core of otolaryngologists and neurosurgeons, the subspecialty has grown dependent on other surgical and nonsurgical specialties that provide guidance during the preoperative planning, assistance during the surgical act, postoperatively differenti- ating the disease process and providing adjuvant therapies. Therefore, the fields of radiology, pathology, anesthesiology, endovascular surgery, plastic and reconstructive surgery, neuro-ophthalmology, endocrinology, medical and radiation oncology, among others, are now embedded with the skull base surgical core seeking a common goal, the best possible care for our patients. Having undergone their respective evolution, these specialties are able to help skull base surgeons in ways that before seemed science fiction.

However, as in other aspects of life, any change in the field of medicine is often met with resistance and criticism. Although constructive discussion and critical appraisal are essential to precipitate desirable adjustments and eliminate ineffective therapies, resistance in the face of factual evidence commonly represents a fear to move out of the individual "comfort zone." As evidence supporting the safety and effectiveness of various minimally invasive and minimal access techniques continue to accumulate and to be reproduced throughout the world, those initial opposing and heated arguments have significantly dissipated. Increasingly, skull base surgeons seek to learn and adopt these techniques.

Technology has also changed the way we disburse and consume new information. Continued medical education, to update clinical and surgical concepts and to learn new techniques, has never been more available and paradoxically so overwhelming. Nonetheless, despite the emergence of various interactive models, e-learning, and simulators, peer-reviewed journals continue to be a pillar of the medical learning process. Following this observation, we offer this special edition of the International Archives of Otorhinolaryngology, in which we have gathered the expert opinions of recognized leaders in several of the fields that currently comprise part of the Skull Base Team. Although by no means comprehensive, this special edition delivers an concise update on computed tomography and magnetic resonance imaging, techniques that form the foundation of our surgical planning and postoperative

Address for correspondence 
surveillance; on advances in surgical techniques addressing sellar and clival lesions, while also providing a historical perspective on the evolution of the transsphenoidal approach; on current concepts regarding the management of catastrophic hemorrhage due to internal carotid artery injury; and on current methods of reconstruction.
I have been honored and humbled by the opportunity conferred by the International Journal of Otorhinolaryngology to serve as a guest editor for this special issue. Moreover, I am extremely grateful and indebted to the authors that selflessly contributed their time and expertise to this edition. 